Lepr Rev (1997) 68, 114-116

\title{
Editorial
}

\section{THE RISK OF RELAPSE AFTER MULTIDRUG THERAPY IN LEPROSY}

The general concept of 'relapse' is the recurrence of a disease after cure. 'Cure' is again a concept which according to the patient or to the treating physician, means relief from the symptoms and the disappearance of the signs. A radical cure envisages, particularly in an infectious disease, a total elimination of the cause, namely the infecting agent. If the cause is totally removed, a recurrence of the disease could only be a reinfection, rather than a relapse. As such, a relapse invariably presumes persistence of the causative agent.

Earlier leprologists were reluctant to use the word 'cure' in leprosy and employed such words as 'quiescent' or 'arrested', not being sure of a total elimination of the causative agent. Today, with the advent of potent bacteriocidal drugs, we are emboldened to declare a case as cured. Even so, it is doubtful whether in highly-bacillated cases, total elimination is possible. It is estimated that there could be $10^{12}$ or $10^{13}$ bacilli in a lepromatous case of leprosy. Even if $99.999 \%$ of the germs are killed by the drug, the remaining $0.001 \%$ of the bacilli would still constitute a significant number. Added to this, there is the question of 'persister' bacilli which remain dormant in tissues into which drugs do not penetrate. The occurrence of drug-resistant or persistent bacilli could cause a relapse.

These are theoretical considerations. However, in practice it is not a significant problem. It is quoted that the relapse rate is well below $1 \%$ with WHO-multidrug therapy (WHOMDT), which has a high degree of efficacy against multibacillary leprosy. ${ }^{1}$ In a question survey organized by $\mathrm{WHO},{ }^{4}$ data obtained from 30 centres from 17 countries showed an overall relapse rate of $0.51 \%$ or 2.3 per 100 patient years. Further data obtained from selected programmes, which maintain excellent information systems, showed that out of 20,141 patients observed during 1984-1992, there were only 67 cases of relapse. The relapse rate during different periods of follow-up being $0.07 \%$ in the first 3 years, $0.09 \%$ during 4-6 years, and $0.07 \%$ during 7-9 years. Probably the small number of bacilli remaining viable after potent chemotherapy are taken care of by the body's general immune system, preventing relapse of the disease.

While these data are encouraging, a recent publication by Jamet et $a l^{3}{ }^{3}$ causes considerable concern. From a group of 35 multibacillary cases treated by WHO-MDT regimen for 2 years, 7 cases of relapse were found during a follow-up ranging from 27 to 84 months, giving a relapse rate of $20 \%$. In an earlier publication from the same group of investigators, ${ }^{2}$ obviously on the same group of patients, the relapse rate with WHO-MDT was only $2.9 \%$ during a follow-up period of 27 months. This indicated that relapses occur late, 5-7 years 
after stopping MDT. It was further found by this group that the relapse rate closely correlated with the bacterial load before and at the end of fixed duration MDT of 24 months. ${ }^{3}$

The observation of Jamet et $a l .{ }^{3}$ and the Marchaux Chemotherapy Study Group ${ }^{2}$ needs careful consideration. The two factors which govern the observed rate of relapse are the high bacterial load in the patients and the long period of follow-up. This has to be considered against the recommendation of $\mathrm{WHO}^{4}$ that, "because the risk of relapse after completion of WHO-MDT regimens has been shown to be negligible, it is no longer necessary to continue routine annual surveillance of patients. The WHO recommendation is based on the low rate of relapse as assessed by their question survey. The data pertains to the group of multibacillary (MB) cases taken as a whole. The high rate of relapse reported by Jamet et al. ${ }^{3}$ is among the highly-bacillated patients. Such patients no doubt form a very small proportion in the field, but if neglected could be a source of infection and possibly a source of drug-resistant strains. $\mathrm{MB}$ cases with initial high BI should be followed up with annual surveillance for a minimum period of 5-7 years.

This raises the question of skin smears in the field. The tendency in most national programmes is to diminish the importance of skin smears or drop them completely on the excuse of the poor standard of many of the field laboratories. Attempts must be made to improve the standard of field laboratories, rather than closing them down and avoiding doing skin smears. Fixed duration therapy is recommended today, and MB cases are released from treatment even if the skin smears are positive at the completion of 2 years of treatment. The findings of Jamet $e t a l{ }^{3}$ indicate that in cases of a bacillary index (BI) of 4.0 before MDT or a $\mathrm{BI}$ of 3.0 at the end of MDT, the risk of relapse is very high. Hence, monitoring the bacterial load in a patient by regular skin smears is essential. At the same time quality control on laboratory services should be maintained with regular monitoring by reference laboratories.

Short course chemotherapy is most welcome in a chronic disease like leprosy. The WHOMDT regimen has proved its efficacy and fixed duration therapy is worth a trial. Further, the newer antileprosy drugs hold a great promise. It is hoped that even more potent drugs would be available in future which may make it possible to cure an MB case in 2-3 months. But it should not be forgotten that Mycobacterium leprae are notorious for remaining dormant in protected tissues for several years. Hence the need for follow-up and careful surveillance remains extremely relevant. The finding of Jamet et $a l^{3}$ that a clinically-cured case could relapse after 5-7 years, particularly if the patient is highly bacillated, is an important lesson for the field programme.

The WHO study ${ }^{5}$ while ruling out the necessity to continue annual surveillance has suggested that patients should be taught to recognize early signs of relapse and report promptly for treatment. Relapse in lepromatous cases is first by bacterial increase and it is difficult for the patient to recognize skin changes. By the time they recognize skin changes and relapse, the bacterial load will have increased considerably with consequent problems. Thus MB cases with high initial BI should get special attention with regard to regular postMDT follow-up and bacterialogical assessment. This will not add greatly to the workload of the field programme because there will be only a small number of such cases.

\section{References}

1 ALM Consensus Development Conference on Chemotherapy of Leprosy. Consensus development statement on chemotherapy of leprosy. Int J Lepr, 1992; 60: 644-652. 
2 Marchoux Chemotherapy Study Group. Relapses in multibacillary leprosy patients after stopping treatment with rifampin-containing combined regimens. Int J Lepr, 1992; 60: 525-535.

3 Jamet P, Ji Baohony and the Marchoux Chemotherapy Study Group. Relapse after long-term follow up of multibacillary patients treated by WHO multidrug regimen. Int J Lepr, 1995; 63: 195-201.

4 WHO Leprosy Unit. Risk of relapse in Leprosy. WHO document WHO/CTD/LEP 94.1.

5 WHO, Action programme for elimination of leprosy. Lep News, 1994; 3: 1-5.

Leprosy Histopathology Centre

K. V. DESIKAN

Mahatma Gandhi Institute of Medical Sciences

Sevagram 442102

India 